\title{
NEW CHARACTERIZATIONS OF THE CLIFFORD TORUS AS A LAGRANGIAN SELF-SHRINKER
}

\author{
HAIZHONG LI AND XIANFENG WANG
}

\begin{abstract}
In this paper, we obtain several new characterizations of the Clifford torus as a Lagrangian self-shrinker. We first show that the Clifford torus $\mathbb{S}^{1}(1) \times \mathbb{S}^{1}(1)$ is the unique compact orientable Lagrangian self-shrinker in $\mathbb{C}^{2}$ with $|A|^{2} \leq 2$, which gives an affirmative answer to Castro-Lerma's conjecture in 6 . We also prove that the Clifford torus is the unique compact orientable embedded Lagrangian self-shrinker with nonnegative or nonpositive Gauss curvature in $\mathbb{C}^{2}$.
\end{abstract}

\section{INTRODUCTION}

Let $x: M^{n} \rightarrow \mathbb{R}^{n+p}$ be an $n$-dimensional submanifold in the $(n+p)$-dimensional Euclidean space. We call the immersed manifold $M^{n}$ a self-shrinker if it satisfies the quasilinear elliptic system:

$$
\mathbf{H}=-x^{\perp}
$$

where $\mathbf{H}$ is the mean curvature vector and $\perp$ denotes the projection onto the normal bundle of $M^{n}$.

Self-shrinkers play an important role in the study of the mean curvature flow. Not only they correspond to self-shrinking solutions to the mean curvature flow, but also they describe all possible Type I blow ups at a given singularity of the mean curvature flow. There are many results about the classification of self-shrinkers. In the curve case, Abresch and Langer [1] gave a complete classification of all solutions to (1.1). These curves are called Abresch-Langer curves. In higher dimension and codimension one, Huisken (see [12] and [13]) proved that $n$-dimensional smooth complete self-shrinkers in $\mathbb{R}^{n+1}$ with $H \geq 0$, polynomial volume growth, and $|A|$ bounded are $\Gamma \times \mathbb{R}^{n-1}$, or $\mathbb{S}^{m}(\sqrt{m}) \times \mathbb{R}^{n-m}(0 \leq m \leq n)$, where $\Gamma$ is an Abresch-Langer curve and $\mathbb{S}^{m}(\sqrt{m})$ is an $m$-dimensional sphere of radius $\sqrt{m}$. In [9], Colding and Minicozzi showed that Huisken's classification holds without the assumption that $|A|$ is bounded.

In arbitrary codimensional case, Smoczyk 24] proved that (i) If $M^{n}$ is a compact selfshrinker in $\mathbb{R}^{n+p}$, then $M^{n}$ is a minimal submanifold of the sphere $\mathbb{S}^{n+p-1}(\sqrt{n})$ if and only if $\mathbf{H} \neq 0$ and $\nabla^{\perp} \nu=0$, where $\nu=\mathbf{H} /|\mathbf{H}|$ is the principal normal. (ii) Let $M^{n}$ be a complete non-compact self-shrinker in $\mathbb{R}^{n+p}$, if $\mathbf{H} \neq 0, \nabla^{\perp} \nu=0$, and $M^{n}$ has uniformly bounded geometry, then $M^{n}$ is either $\Gamma \times \mathbb{R}^{n-1}$ or $N^{m} \times \mathbb{R}^{n-m}$, where $\Gamma$ is an AbreschLanger curve and $N^{m}$ is an $m$-dimensional complete minimal submanifold in $\mathbb{S}^{m+p-1}(\sqrt{m})$. In [18, using the method of Colding and Minicozzi [9], Li and Wei showed that Smoczyk's result in complete non-compact case holds under a weaker condition.

2010 Mathematics Subject Classification. primary 53C44; secondary 53D12.

Key words and phrases. Lagrangian self-shrinker, rigidity, Clifford torus, mean curvature flow, Gauss curvature.

The first author was supported in part by NSFC Grant No. 11271214. The second author was supported in part by NSFC (Grant Nos. 11201243 and 11571185) and "Specialized Research Fund for the Doctoral Program of Higher Education, Grant No. 20120031120026". 
We recall some rigidity theorems for self-shrinkers. The first gap of the squared norm of the second fundamental form $|A|^{2}$ for self-shrinkers was obtained by Cao and $\mathrm{Li}$ [4] (which generalized codimension one case in [14]), they proved that if $M^{n}$ is a complete self-shrinker in $\mathbb{R}^{n+p}$, with polynomial volume growth and satisfying $|A|^{2} \leq 1$, then either $|A|^{2}=0$ and $M^{n}$ is a hyperplane $\mathbb{R}^{n}$, or $|A|^{2}=1$ and $M^{n}$ is a round sphere $\mathbb{S}^{n}(\sqrt{n})$ or a cylinder $\mathbb{S}^{m}(\sqrt{m}) \times \mathbb{R}^{n-m}(1 \leq m \leq n-1)$. Cheng and Peng [7] obtained some rigidity theorems on complete self-shrinkers without assumption on polynomial volume growth. Ding and Xin [10] studied the second gap of $|A|^{2}$ for self-shrinkers in codimension one, they showed that if $M^{n}$ is a complete self-shrinkers in $\mathbb{R}^{n+1}$, with polynomial volume growth and satisfying $1 \leq|A|^{2} \leq 1+0.022$, then $|A|^{2}=1$. Cheng and Wei [8] improved the pinching constant 0.022 to $3 / 7$ under the assumption that $|A|^{2}$ is constant.

In this paper, we are interested in rigidity results for compact Lagrangian self-shrinkers in $\mathbb{C}^{2}$. An immersed manifold $M^{n}$ in $\mathbb{C}^{n}$ is called a Lagrangian submanifold if the standard complex structure $J$ of $\mathbb{C}^{n}$ maps each tangent space of $M^{n}$ into its corresponding normal space. A Lagrangian submanifold $M^{n}$ in $\mathbb{C}^{n}$ is called a Lagrangian self-shrinker if it satisfies (1.1). Recently, the study of Lagrangian self-shrinkers has drawn some attentions. For instance, many examples of Lagrangian self-shrinkers in $\mathbb{C}^{n}$ were constructed in [2, [5] and [15, Hamiltonian stationary Lagrangian self-shrinkers in $\mathbb{C}^{2}$ were classified in [5]. The canonical example of a compact Lagrangian self-shrinker in $\mathbb{C}^{2}$ is the Clifford torus $\mathbb{S}^{1}(1) \times \mathbb{S}^{1}(1)$, which is the standard example of monotone Lagrangian in $\mathbb{C}^{2}$ (see [21]). In [6], Castro and Lerma obtained the following rigidity result for the Clifford torus.

Theorem 1.1 (see Theorem 1.2 in [6]). Let $x: M^{2} \rightarrow \mathbb{C}^{2}$ be a compact orientable Lagrangian self-shrinker. If $|A|^{2} \leq 2$, then $|A|^{2}=2$ and $M^{2}$ is a topological torus. If, in addition, the Gauss curvature $K$ of $M^{2}$ is nonnegative or nonpositive, then $M^{2}$ is the Clifford torus $\mathbb{S}^{1}(1) \times \mathbb{S}^{1}(1)$.

Castro and Lerma conjectured (see page 1519 in [6]) that the condition "the Gauss curvature $K$ of $M^{2}$ is nonnegative or nonpositive " is unnecessary in Theorem 1.1. Our following Theorem 1.2 gives an affirmative answer to their conjecture. In fact, in Section 4 , we prove

Theorem 1.2. Let $x: M^{2} \rightarrow \mathbb{C}^{2}$ be a compact orientable Lagrangian self-shrinker. If $|A|^{2} \leq 2$, then $|A|^{2}=2$ and $M^{2}$ is the Clifford torus $\mathbb{S}^{1}(1) \times \mathbb{S}^{1}(1)$.

Remark 1.3. For any $m, n \in \mathbb{N},(m, n)=1, m \leq n$, Lee and Wang [15] constructed the following example $T_{m, n}$ of Lagrangian self-shrinker by

$$
\Psi_{m, n}: \mathbb{R}^{2} \rightarrow \mathbb{C}^{2},(s, t) \longmapsto \sqrt{m+n}\left(\frac{1}{\sqrt{n}} \cos s e^{i \sqrt{\frac{n}{m}} t}, \frac{1}{\sqrt{m}} \sin s e^{i \sqrt{\frac{m}{n}} t}\right),
$$

with the squared norm of the second fundamental form satisfying $\frac{3 m^{2}+n^{2}}{n(m+n)} \leq|A|^{2} \leq \frac{m^{2}+3 n^{2}}{m(m+n)}$ (cf. [6]). $\forall \epsilon>0$, if $m$ and $n$ are integers satisfying that $m>\frac{3}{\epsilon}$ and $n=m+1$, then $(m, n)=1$ and $|A|^{2} \leq \frac{m^{2}+3 n^{2}}{m(m+n)}<2+\epsilon$, so there exist infinitely many examples $T_{m, n}$ satisfying $|A|^{2} \leq 2+\epsilon$. In other words, Lee-Wang's examples $T_{m, n}$ have an upper bound on $|A|^{2}$ which gets arbitrarily close to 2 . This shows that the pinching constant 2 is optimal in Theorem 1.2 .

In the last section, we prove the following classification theorem for compact orientable Lagrangian self-shrinker in $\mathbb{C}^{2}$ with nonnegative Gauss curvature.

Theorem 1.4. Let $x: M^{2} \rightarrow \mathbb{C}^{2}$ be a compact orientable Lagrangian self-shrinker. If the Gauss curvature $K$ of $M^{2}$ is nonnegative, then $K=0$ and $M^{2}$ is the Riemannian product of two closed Abresch-Langer curves. 
If $x: M^{2} \rightarrow \mathbb{C}^{2}$ is embedded, using the result of Abresch-Langer which states that the only closed embedded self-shrinker in $\mathbb{R}^{2}$ is the circle, as an immediate consequence of Theorem 1.4, we obtain the following new characterization of the Clifford torus $\mathbb{S}^{1}(1) \times$ $\mathbb{S}^{1}(1)$.

Corollary 1.5. The Clifford torus $\mathbb{S}^{1}(1) \times \mathbb{S}^{1}(1)$ is the unique compact orientable embedded Lagrangian self-shrinker in $\mathbb{C}^{2}$ with nonnegative Gauss curvature.

Remark 1.6. In [22], Neves proposed the following question (see Question 7.4 in [22]): Find a condition on a Lagrangian torus in $\mathbb{C}^{2}$, which implies that Lagrangian mean curvature flow $\left(L_{t}\right)_{0<t<T}$ will become extinct at time $T$ and, after rescale, $L_{t}$ converges to the Clifford torus. Our new characterizations of the Clifford torus might be useful to this question.

Remark 1.7. We note that in Theorem 1.2, one does not need to assume that the Lagrangian self-shrinker is embedded, but we need this for Corollary 1.5. We also note that the conclusion is still true if one replaces the assumption "nonnegative Gauss curvature" by "nonpositive Gauss curvature" in Corollary 1.5, see Corollary 5.9 for more details.

The paper is organized as follows: in the next section, we recall some basic formulas for Lagrangian submanifolds of $\mathbb{C}^{2}$. In Section 3, we give some identities and lemmas for Lagrangian self-shrinkers of $\mathbb{C}^{2}$. In Section 4 , we prove Theorem 1.2, we also prove that the Clifford torus $\mathbb{S}^{1}(1) \times \mathbb{S}^{1}(1)$ is the unique compact orientable Lagrangian self-shrinker in $\mathbb{C}^{2}$ with $|A|^{2}$ being constant, which is the key step of the proof of Theorem 1.2. In Section 5, we prove Theorem 1.4. Throughout this paper, we always assume that $M$ is connected and has no boundary.

\section{Preliminaries}

In this section, $M^{2}$ will always denote a 2-dimensional Lagrangian submanifold of $\mathbb{C}^{2}$. We denote the Levi-Civita connections on $M^{2}, \mathbb{C}^{2}$ and the normal bundle by $\nabla, D$ and $\nabla^{\perp}$, respectively. The formulas of Gauss and Weingarten are given by

$$
D_{X} Y=\nabla_{X} Y+h(X, Y), D_{X} \xi=-A_{\xi} X+\nabla_{X}^{\perp} \xi
$$

where $h$ is the second fundamental form, A denotes the shape operator, $X$ and $Y$ are tangent vector fields and $\xi$ is a normal vector field on $M^{2}$.

The Lagrangian condition implies that (cf. [16], [17])

$$
\nabla_{X}^{\perp} J Y=J \nabla_{X} Y, A_{J X} Y=-J h(X, Y)=A_{J Y} X .
$$

The formulas above imply that $\langle h(X, Y), J Z\rangle$ is totally symmetric, i.e.,

$$
\langle h(X, Y), J Z\rangle=\langle h(Y, Z), J X\rangle=\langle h(Z, X), J Y\rangle,
$$

where $\langle$,$\rangle denotes the standard inner product in \mathbb{C}^{2}$.

For a Lagrangian submanifold $M^{2}$ in $\mathbb{C}^{2}$, an orthonormal frame field

$$
e_{1}, e_{2}, e_{1^{*}}, e_{2^{*}}
$$

is called an adapted Lagrangian frame field if $e_{1},, e_{2}$ are orthonormal tangent vector fields and $e_{1^{*}}, e_{2^{*}}$ are normal vector fields given by

$$
e_{1^{*}}=J e_{1}, e_{2^{*}}=J e_{2} .
$$

The dual frame fields of $e_{1}, e_{2}$ are $\theta_{1}, \theta_{2}$, the Levi-Civita connection forms and normal connection forms are $\theta_{i j}$ and $\theta_{i^{*} j^{*}}$, respectively. Writing $h\left(e_{i}, e_{j}\right)=\sum_{k} h_{i j}^{k^{*}} e_{k^{*}}$, (2.3) is 
equivalent to

$$
h_{i j}^{k^{*}}=h_{j k}^{i^{*}}=h_{k i}^{j^{*}}, 1 \leq i, j, k \leq 2 .
$$

We have the following structure equations.

$$
\begin{gathered}
d x=\sum_{i} \theta_{i} e_{i} \\
d e_{i}=\sum_{j} \theta_{i j} e_{j}+\sum_{j, k} h_{i j}^{k^{*}} \theta_{j} e_{k^{*}}, \\
d e_{k^{*}}=-\sum_{i, j} h_{i j}^{k^{*}} \theta_{j} e_{i}+\sum_{l} \theta_{k^{*} l^{*}} e_{l^{*}}
\end{gathered}
$$

If we denote the components of curvature tensors of $\nabla$ and $\nabla^{\perp}$ by $R_{i j k l}$ and $R_{i^{*} j^{*} k l}$, respectively, then the equations of Gauss, Codazzi and Ricci are given by (cf. [16], [17])

$$
\begin{gathered}
R_{m i l p}=\sum_{j}\left(h_{m l}^{j^{*}} h_{i p}^{j^{*}}-h_{m p}^{j^{*}} h_{i l}^{j^{*}}\right), \\
R_{j k}=\sum_{p} H^{p^{*}} h_{j k}^{p^{*}}-\sum_{i, p} h_{i j}^{p^{*}} h_{i k}^{p^{*}}, \\
h_{i j, l}^{k^{*}}=h_{i l, j}^{k^{*}}, 1 \leq i, j, k, l \leq 2, \\
R_{i^{*} j^{*} k l}=\sum_{m}\left(h_{m k}^{i^{*}} h_{m l}^{j^{*}}-h_{m l}^{i^{*}} h_{m k}^{j^{*}}\right), \\
R=H^{2}-|A|^{2},
\end{gathered}
$$

where $R_{j k}$ and $R$ are the Ricci curvature and the scalar curvature of $M^{2}$, respectively, $|A|^{2}=\sum_{i, j, k}\left(h_{i j}^{k^{*}}\right)^{2}$ is the squared norm of the second fundamental form, $\mathbf{H}=\sum_{k} H^{k^{*}} e_{k^{*}}=$ $\sum_{i, k} h_{i i}^{k^{*}} e_{k^{*}}$ is the mean curvature vector field, $H=|\mathbf{H}|$ is the mean curvature of $M^{2}$, and $h_{i j, l}^{k^{*}}$ is defined by

$$
\sum_{l} h_{i j, l}^{k^{*}} \theta_{l}=d h_{i j}^{k^{*}}+\sum_{l} h_{l j}^{k^{*}} \theta_{l i}+\sum_{l} h_{i l}^{k^{*}} \theta_{l j}+\sum_{m} h_{i j}^{m^{*}} \theta_{m^{*} k^{*}}
$$

We can write (2.14) in the following equivalent form:

$$
\left(\nabla_{X} h\right)(Y, Z)=\nabla_{X}^{\perp} h(Y, Z)-h\left(\nabla_{X} Y, Z\right)-h\left(Y, \nabla_{X} Z\right),
$$

where $X, Y$ and $Z$ are tangent vector fields on $M^{2}$. We note that $\left(\nabla_{e_{k}} h\right)\left(e_{i}, e_{j}\right)=$ $\sum_{l} h_{i j, k}^{l^{*}} e^{*}$.

Combining (2.5) and (2.11), we know that $h_{i j, l}^{k^{*}}$ is totally symmetric, i.e.,

$$
h_{i j, l}^{k^{*}}=h_{j l, k}^{i^{*}}=h_{l k, i}^{j^{*}}=h_{k i, j}^{l^{*}}, 1 \leq i, j, k, l \leq 2 .
$$

We have the following Ricci identities.

$$
h_{i j, l p}^{k^{*}}-h_{i j, p l}^{k^{*}}=\sum_{m} h_{m j}^{k^{*}} R_{m i l p}+\sum_{m} h_{i m}^{k^{*}} R_{m j l p}+\sum_{m} h_{i j}^{m^{*}} R_{m^{*} k^{*} l p},
$$

where $h_{i j, l p}^{k^{*}}$ is defined by

$$
\sum_{p} h_{i j, l p}^{k^{*}} \theta_{p}=d h_{i j, l}^{k^{*}}+\sum_{p} h_{p j, l}^{k^{*}} \theta_{p i}+\sum_{p} h_{i p, l}^{k^{*}} \theta_{p j}+\sum_{p} h_{i j, p}^{k^{*}} \theta_{p l}+\sum_{p} h_{i j, l}^{p^{*}} \theta_{p^{*} k^{*}} .
$$


Using (2.5), (2.9) and (2.12), we have

$$
R_{m^{*} i^{*} l p}=R_{m i l p}
$$

We define the first and second covariant derivatives, and Laplacian of the mean curvature vector field $\mathbf{H}=\sum_{k} H^{k^{*}} e_{k^{*}}$ in the normal bundle $N\left(M^{2}\right)$ as follows.

$$
\begin{gathered}
\sum_{i} H_{, i}^{k^{*}} \theta_{i}=d H^{k^{*}}+\sum_{l} H^{l^{*}} \theta_{l^{*} k^{*}} \\
\sum_{j} H_{, i j}^{k^{*}} \theta_{j}=d H_{, i}^{k^{*}}+\sum_{j} H_{, j}^{k^{*}} \theta_{j i}+\sum_{l} H_{, i}^{l^{*}} \theta_{l^{*} k^{*}} \\
\Delta^{\perp} H^{k^{*}}=\sum_{i} H_{, i i}^{k^{*}}, H^{k^{*}}=\sum_{i} h_{i i}^{k^{*}} .
\end{gathered}
$$

Let $f$ be a smooth function on $M^{2}$, we define the covariant derivatives $f_{, i}, f_{, i j}$, and the Laplacian of $f$ as follows.

$$
d f=\sum_{i} f_{, i} \theta_{i}, \sum_{j} f_{, i j} \theta_{j}=d f_{, i}+\sum_{j} f_{, j} \theta_{j i}, \Delta f=\sum_{i} f_{, i i} .
$$

\section{Some Identities And Lemmas}

In this section, we assume that $x: M^{2} \rightarrow \mathbb{C}^{2}$ is a compact orientable Lagrangian self-shrinker. The self-shrinker equation (1.1) is equivalent to

$$
H^{k^{*}}=-\left\langle x, e_{k^{*}}\right\rangle, 1 \leq k \leq 2
$$

Lemma 3.1 (cf. [4]). Let $x: M^{2} \rightarrow \mathbb{C}^{2}$ be a Lagrangian self-shrinker, we have

$$
\begin{gathered}
H_{, i}^{k^{*}}=\sum_{j} h_{i j}^{k^{*}}\left\langle x, e_{j}\right\rangle, 1 \leq i, k \leq 2, \\
H_{, i j}^{k^{*}}=\sum_{m} h_{i m, j}^{k^{*}}\left\langle x, e_{m}\right\rangle+h_{i j}^{k^{*}}-\sum_{m, p} H^{p^{*}} h_{i m}^{k^{*}} h_{m j}^{p^{*}}, 1 \leq i, j, k \leq 2 .
\end{gathered}
$$

Proof. From (2.23) and the structure equations (2.6)-(2.8), we obtain

$$
x_{, i}=e_{i}, x_{, i j}=e_{i, j}=\sum_{k} h_{i j}^{k^{*}} e_{k^{*}}
$$

and

$$
e_{k^{*}, i}=-\sum_{j} h_{i j}^{k^{*}} e_{j}, e_{k^{*}, i j}=-\sum_{m} h_{i m, j}^{k^{*}} e_{m}-\sum_{m, p} h_{i m}^{k^{*}} h_{m j}^{p^{*}} e_{p^{*}} .
$$

Taking covariant derivative of (3.1) with respect to $e_{i}$ by use of (3.4) and (3.5), we obtain (3.2). Taking covariant derivative of (3.2) with respect to $e_{j}$ by use of (3.4) and (3.1), we obtain (3.3).

Recall the following operator $\mathcal{L}$ which was introduced and studied firstly on self-shrinkers by Colding and Minicozzi (see (3.7) in [9]): $\mathcal{L}=\Delta-\langle x, \nabla \cdot\rangle=e^{|x|^{2} / 2} \operatorname{div}\left(e^{-|x|^{2} / 2} \nabla \cdot\right)$, where $\Delta, \nabla$ and div denote the Laplacian, gradient and divergent operator on the self-shrinker, respectively. The operator $\mathcal{L}$ is self-adjoint in a weighted $L^{2}$ space. 
Lemma 3.2. Let $x: M^{2} \rightarrow \mathbb{C}^{2}$ be a Lagrangian self-shrinker, we have

$$
\frac{1}{2} \mathcal{L}|A|^{2}=|\nabla A|^{2}+|A|^{2}-\frac{3}{2}|A|^{4}+2 H^{2}|A|^{2}-\frac{1}{2} H^{4}-\sum_{i, j, k, l} H^{k^{*}} H^{l^{*}} h_{i j}^{k^{*}} h_{i j}^{l^{*}} .
$$

Proof. By definition of $\Delta$ and using (2.9), (2.12), (2.16), (2.17), (2.19) and (3.3), we have

$$
\begin{aligned}
& \frac{1}{2} \Delta|A|^{2}=\sum_{i, j, k, p}\left(h_{i j, k}^{p^{*}}\right)^{2}+\sum_{i, j, k, p} h_{i j}^{p^{*}} h_{i j, k k}^{p^{*}} \\
= & |\nabla A|^{2}+\sum_{i, j, k, p} h_{i j}^{p^{*}} h_{k k, i j}^{p^{*}}+\sum_{i, j, m, p} h_{i j}^{p^{*}} h_{m i}^{p^{*}} R_{m j}+\sum_{i, j, k, m, p} h_{i j}^{p^{*}} h_{k m}^{p^{*}} R_{m i j k}+\sum_{i, j, k, m, p} h_{i j}^{p^{*}} h_{k i}^{m^{*}} R_{m^{*} p^{*} j k} \\
= & |\nabla A|^{2}+\sum_{k} \frac{1}{2}\left(|A|^{2}\right)_{, k}\left\langle x, e_{k}\right\rangle+|A|^{2}-\sum_{i, j, k, m, p} H^{m^{*}} h_{i j}^{p^{*}} h_{i k}^{p^{*}} h_{k j}^{m^{*}} \\
& +\sum_{i, j, m, p} h_{i j}^{p^{*}} h_{m i}^{p^{*}} R_{m j}+\sum_{i, j, k, m, p} h_{i j}^{p^{*}} h_{k m}^{p^{*}} R_{m i j k}+\sum_{i, j, k, m, p} h_{i j}^{p^{*}} h_{k i}^{m^{*}} R_{m^{*} p^{*} j k} .
\end{aligned}
$$

Since $M^{2}$ is a Lagrangian surface in $\mathbb{C}^{2}$, denote the Gauss curvature of $M^{2}$ by $K$, from (2.9) and (2.19), we have

$$
\begin{aligned}
& -\sum_{i, p} h_{i j}^{p^{*}} h_{i k}^{p^{*}}=K \delta_{j k}-\sum_{p} H^{p^{*}} h_{j k}^{p^{*}}, \\
& R_{m i j k}=K\left(\delta_{m j} \delta_{i k}-\delta_{m k} \delta_{i j}\right), R_{m^{*} p^{*} j k}=K\left(\delta_{m j} \delta_{p k}-\delta_{m k} \delta_{p j}\right),
\end{aligned}
$$

substituting (3.8) into (3.7), using Gauss equation (2.13), we obtain

$$
\frac{1}{2} \mathcal{L}|A|^{2}=|\nabla A|^{2}+|A|^{2}-\frac{3}{2}|A|^{4}+2 H^{2}|A|^{2}-\frac{1}{2} H^{4}-\sum_{i, j, k, l} H^{k^{*}} H^{l^{*}} h_{i j}^{k^{*}} h_{i j}^{l^{*}}
$$

Lemma 3.3 (cf. [4], 9]). Let $x: M^{2} \rightarrow \mathbb{C}^{2}$ be a compact orientable Lagrangian selfshrinker, we have

$$
\begin{aligned}
& 0=\int_{M}^{2} \frac{1}{2} \Delta\left(|x|^{2}\right) d v=\int_{M}^{2}\left(2-H^{2}\right) d v \\
& 0=\int_{M}^{2} \frac{1}{2} \mathcal{L}\left(|x|^{2}\right) e^{-\frac{|x|^{2}}{2}} d v=\int_{M}^{2}\left(2-|x|^{2}\right) e^{-\frac{|x|^{2}}{2}} d v .
\end{aligned}
$$

Proof. It follows from (3.4) and (3.1) that

$$
\begin{gathered}
\frac{1}{2} \Delta\left(|x|^{2}\right)=2+\langle x, \Delta x\rangle=2+\sum_{k} H^{k^{*}}\left\langle x, e_{k^{*}}\right\rangle=2-H^{2}, \text { which implies that } \\
0=\int_{M}^{2} \frac{1}{2} \Delta\left(|x|^{2}\right) d v=\int_{M}^{2}\left(2-H^{2}\right) d v \text { and } \frac{1}{2} \mathcal{L}\left(|x|^{2}\right)=\frac{1}{2} \Delta\left(|x|^{2}\right)-\sum_{i}\left\langle x, e_{i}\right\rangle^{2}=2-|x|^{2} .
\end{gathered}
$$

The last equation in (3.10) follows from the fact that the operator $\mathcal{L}$ is self-adjoint in a weighted $L^{2}$ space.

\section{Proof of Theorem 1.2}

In this section, we prove Theorem 1.2. First, we recall the following lemma which is important in the proof of our key Proposition 4.2. It was proved in [6] by Castro and Lerma by using Gauss-Bonnet theorem combined with the Gauss equation in a clever way. 
Lemma 4.1 (see Theorem 1.2 in [6]). Let $x: M^{2} \rightarrow \mathbb{C}^{2}$ be a compact orientable Lagrangian self-shrinker. If $|A|^{2} \leq 2$, then $|A|^{2}=2$ and $M^{2}$ is a topological torus.

Proof. Denote the Gauss curvature of $M^{2}$ by $K$. From Gauss equation $R=2 K=H^{2}-|A|^{2}$ and Gauss-Bonnet theorem, we have

$$
8 \pi\left(1-\operatorname{gen}\left(M^{2}\right)\right)=2 \int_{M}^{2} K d v=\int_{M}^{2}\left(H^{2}-|A|^{2}\right) d v=\int_{M}^{2}\left(2-|A|^{2}\right) d v,
$$

where gen $\left(M^{2}\right)$ stands for the genus of $M^{2}$ and the last equality is due to Lemma 3.3 . It is well known that there exist no Lagrangian self-shrinkers in $\mathbb{C}^{n}$ with the topology of sphere, which was proved by Smoczyk (see [23], Theorem 2.3.5, see also Theorem 2.1 in [6] for a detailed proof). Hence, if $|A|^{2} \leq 2$, then $|A|^{2}=2$ and $M^{2}$ is a topological torus.

We are now ready to prove the following key proposition:

Proposition 4.2. Let $x: M^{2} \rightarrow \mathbb{C}^{2}$ be a compact orientable Lagrangian self-shrinker. If the squared norm of the second fundamental form $|A|^{2}$ is constant, then $|A|^{2}=2$ and $M^{2}$ is the Clifford torus $\mathbb{S}^{1}(1) \times \mathbb{S}^{1}(1)$.

Proof. We prove by two steps. Firstly, we show that $|A|^{2}=2$.

Since $M^{2}$ is compact, there exists a point $p_{0} \in M^{2}$ such that $|x|^{2}$ attains its minimum at $p_{0}$. We immediately have $\left(|x|^{2}\right)_{, j}=0,1 \leq j \leq 2$ at $p_{0}$, which implies that $\left\langle x, e_{j}\right\rangle\left(p_{0}\right)=$ $0,1 \leq j \leq 2$. Hence at $p_{0}$, from (3.1) and (3.2) we have $x=-\mathbf{H},|x|^{2}=H^{2}, H_{, i}^{k^{*}}=$ $0,1 \leq i, k \leq 2$, which lead to the following equations:

$$
h_{11,1}^{1^{*}}+h_{22,1}^{1^{*}}=0, h_{11,2}^{1^{*}}+h_{22,2}^{1^{*}}=0, h_{11,2}^{2^{*}}+h_{22,2}^{2^{*}}=0 .
$$

On the other hand, since $|A|^{2}=\left(h_{11}^{1^{*}}\right)^{2}+3\left(h_{12}^{1^{*}}\right)^{2}+3\left(h_{12}^{2^{*}}\right)^{2}+\left(h_{22}^{2^{*}}\right)^{2}$ is constant, we have $\left(|A|^{2}\right)_{, k}=0,1 \leq k \leq 2$. Therefore,

$$
\begin{aligned}
& h_{11}^{1^{*}} h_{11,1}^{1^{*}}+3 h_{12}^{1^{*}} h_{12,1}^{1^{*}}+3 h_{12}^{2^{*}} h_{12,1}^{2^{*}}+h_{22}^{2^{*}} h_{22,1}^{2^{*}}=0 \\
& h_{11}^{1^{*}} h_{11,2}^{1^{*}}+3 h_{12}^{1^{*}} h_{12,2}^{1^{*}}+3 h_{12}^{2^{*}} h_{12,2}^{2^{*}}+h_{22}^{2^{*}} h_{22,2}^{2^{*}}=0 .
\end{aligned}
$$

From (4.2), using (2.16), we get

$$
h_{22,1}^{1^{*}}=-h_{11,1}^{1^{*}}, h_{22,2}^{1^{*}}=-h_{11,2}^{1^{*}}, h_{22,2}^{2^{*}}=h_{11,1}^{1^{*}} .
$$

Since $h_{i j}^{k^{*}}$ and $h_{i j, l}^{k^{*}}$ are both totally symmetric (see (2.5) and (2.16)), by substituting (4.4) into (4.3), we obtain

$$
\begin{aligned}
& \left(h_{11}^{1^{*}}-3 h_{22}^{1^{*}}\right) h_{11,1}^{1^{*}}-\left(h_{22}^{2^{*}}-3 h_{11}^{2^{*}}\right) h_{11,2}^{1^{*}}=0, \\
& \left(h_{22}^{2^{*}}-3 h_{11}^{2^{*}}\right) h_{11,1}^{1^{*}}+\left(h_{11}^{1^{*}}-3 h_{22}^{1^{*}}\right) h_{11,2}^{1^{*}}=0 .
\end{aligned}
$$

Taking the sum of the square of (4.5) and the square of (4.6), we get

$$
\left[\left(h_{11}^{1^{*}}-3 h_{22}^{1^{*}}\right)^{2}+\left(h_{22}^{2^{*}}-3 h_{11}^{2^{*}}\right)^{2}\right]\left[\left(h_{11,1}^{1^{*}}\right)^{2}+\left(h_{11,2}^{1^{*}}\right)^{2}\right]=0 .
$$

Hence, from (4.7), we have the following two possibilities:

(i) At $p_{0}, h_{11}^{1^{*}}=3 h_{22}^{1^{*}}, h_{22}^{2^{*}}=3 h_{11}^{2^{*}}$. In this case, $|A|^{2}\left(p_{0}\right)=\frac{4}{3}\left(\left(h_{11}^{1^{*}}\right)^{2}+\left(h_{22}^{2^{*}}\right)^{2}\right), H^{2}\left(p_{0}\right)=$ $\frac{16}{9}\left(\left(h_{11}^{1^{*}}\right)^{2}+\left(h_{22}^{2^{*}}\right)^{2}\right)$. From Lemma 3.3. as $|x|^{2}$ attains its minimum at $p_{0}$, we obtain that $H^{2}\left(p_{0}\right)=|x|^{2}\left(p_{0}\right) \leq 2$, hence $|A|^{2}=|A|^{2}\left(p_{0}\right)=\frac{3}{4} H^{2}\left(p_{0}\right) \leq \frac{3}{2}$, which is impossible by Lemma 4.1 .

(ii) At $p_{0}, h_{11,1}^{1^{*}}=h_{11,2}^{1^{*}}=0$. Then from (4.4), we have $h_{22,1}^{1^{*}}=h_{22,2}^{1^{*}}=h_{22,2}^{2^{*}}=0$. In this case, $|\nabla A|=0$ at $p_{0}$. By Lemma 3.2, $|A|^{2}-\frac{3}{2}|A|^{4}+2 H^{2}|A|^{2}-\frac{1}{2} H^{4}-\sum_{i, j, k, l} H^{k^{*}} H^{l^{*}} h_{i j}^{k^{*}} h_{i j}^{l^{*}}=$ 
0 at $p_{0}$. If $H\left(p_{0}\right)=0$, then we have $|A|^{2}-\frac{3}{2}|A|^{4}=0$, which implies that $|A|^{2}=0$ or $|A|^{2}=\frac{2}{3}$. By Lemma 4.1, this can not occur, so we have $H\left(p_{0}\right) \neq 0$. Since at $p_{0}, \mathbf{H} \neq 0$, we choose local orthonormal frame $\left\{e_{1}, e_{2}\right\}$ such that $e_{1} / / J \mathbf{H}$ and $H^{1^{*}}=H, H^{2^{*}}=0$, then at $p_{0}$, we have

$$
\begin{aligned}
|A|^{2}-\frac{1}{2}|A|^{4} & =|A|^{4}-2 H^{2}|A|^{2}+\frac{1}{2} H^{4}+\sum_{i, j} H^{2}\left(h_{i j}^{1^{*}}\right)^{2} \\
& =\left(|A|^{2}-H^{2}\right)^{2}+H^{2} \sum_{i, j}\left(h_{i j}^{1^{*}}-\frac{1}{2} H \delta_{i j}\right)^{2} \geq 0,
\end{aligned}
$$

which implies that $|A|^{2}=|A|^{2}\left(p_{0}\right) \leq 2$. Using Lemma 4.1, we know that $|A|^{2} \equiv 2$.

Secondly, we show that $M^{2}$ is the Clifford torus $\mathbb{S}^{1}(1) \times \mathbb{S}^{1}(1)$.

From the arguments above, we know that $|\nabla A|=0$ at $p_{0}$. Moreover, since $|A|^{2}=$ $2=\frac{1}{2}|A|^{4}$, from (4.8), we know that $\left(|A|^{2}-H^{2}\right)^{2}+H^{2} \sum_{i, j}\left(h_{i j}^{1^{*}}-\frac{1}{2} H \delta_{i j}\right)^{2}=0$ at $p_{0}$, which immediately implies that $|A|^{2}=H^{2}$ and $h_{i j}^{1^{*}}=\frac{1}{2} H \delta_{i j}$ at $p_{0}$, we also know that $H^{1^{*}}=h_{11}^{1^{*}}+h_{22}^{1^{*}}=H, H^{2^{*}}=h_{11}^{2^{*}}+h_{22}^{2^{*}}=0$ at $p_{0}$, hence we get that $h_{11}^{1^{*}}=h_{22}^{1^{*}}=\frac{1}{2} H$ and $h_{11}^{2^{*}}=h_{22}^{2^{*}}=0$ at $p_{0}$. Therefore, we have $2=|A|^{2}=|A|^{2}\left(p_{0}\right)=H^{2}\left(p_{0}\right)=|x|^{2}\left(p_{0}\right)$. Since $|x|^{2}$ attains its minimum at $p_{0}$, we get $|x|^{2} \geq 2$, which together with Lemma 3.3 imply that $|x|^{2} \equiv 2$.

Since $|x|^{2} \equiv 2$, we have that $\left\langle x, e_{i}\right\rangle=0, i=1,2$, which means that the position vector $x$ is equal to $x^{\perp}$. Using the self-shrinker equation (1.1), we immediately have that $\mathbf{H}=-x$ and $H^{2}=|x|^{2} \equiv 2$. In particular, we get that $H=|\mathbf{H}|=\sqrt{2}$ and from (2.1) we have

$$
\nabla_{e_{i}}^{\perp} \mathbf{H}=D_{e_{i}} \mathbf{H}+A_{\mathbf{H}} e_{i}=D_{e_{i}}(-x)+A_{\mathbf{H}} e_{i}=-e_{i}+A_{\mathbf{H}} e_{i}, i=1,2,
$$

where in the last equality we use the fact that $x$ is the position vector. In the equation above, $\nabla_{e_{i}}^{\perp} \mathbf{H}$ is a normal vector, $-e_{i}+A_{\mathbf{H}} e_{i}$ is a tangent vector, we get that both of them have to vanish, so we obtain that $\mathbf{H}$ is a non-null parallel normal vector field and hence $J \mathbf{H}$ is a non-null parallel tangent vector field on $M^{2}$. We have also shown that $|A|^{2}=2$. It follows that $H_{, i}^{k^{*}}=0,\left(|A|^{2}\right)_{, k}=0,1 \leq i, k \leq 2$, which means that both (4.2) and (4.3) hold at $\forall p \in M^{2}$. Thus, $\forall p \in M^{2}$, using an analogous argument to that in the first step of the proof, there are two possibilities. (i) At $p, h_{11}^{1^{*}}=3 h_{22}^{1^{*}}, h_{22}^{2^{*}}=3 h_{11}^{2^{*}}$. In this case, $|A|^{2}=\frac{3}{4} H^{2}$, which is a contradiction with $|A|^{2}=H^{2}=2$. (ii) At $p$, $h_{11,1}^{1^{*}}=h_{11,2}^{1^{*}}=h_{22,1}^{1^{*}}=h_{22,2}^{1^{*}}=h_{22,2}^{2^{*}}=0$. Hence, we obtain that $|\nabla A|=0, \forall p \in M^{2}$.

Since $H=\sqrt{2} \neq 0$, we choose local orthonormal frame $\left\{e_{1}, e_{2}\right\}$ such that $e_{1} / / J \mathbf{H}$ and $H^{1^{*}}=H, H^{2^{*}}=0$. As $|\nabla A|=0$, we get that (4.8) holds at $\forall p \in M^{2}$. As $|A|^{2}=2=\frac{1}{2}|A|^{4}$, from (4.8), we know that $\left(|A|^{2}-H^{2}\right)^{2}+H^{2} \sum_{i, j}\left(h_{i j}^{1^{*}}-\frac{1}{2} H \delta_{i j}\right)^{2}=0$, we also know that $H^{1^{*}}=H, H^{2^{*}}=0$, hence under the orthonormal frame $\left\{e_{1}, e_{2}\right\}$ chosen above, we have $h_{11}^{1^{*}}=\frac{\sqrt{2}}{2}, h_{22}^{1^{*}}=\frac{\sqrt{2}}{2}, h_{11}^{2^{*}}=0, h_{22}^{2^{*}}=0$.

In the following, we will determine the explicit expression of the immersion $x$, up to an isometry of $\mathbb{C}^{2}$. Since $H=\sqrt{2}$ is constant and $J \mathbf{H}$ is a non-null parallel tangent vector field on $M^{2}$, we get that $e_{1}$ is parallel on $M^{2}$, hence $\nabla_{e_{i}} e_{j}=0, i, j=1,2$. Therefore, there exist local coordinates $\{u, v\}$ such that $e_{1}=\frac{\partial}{\partial u}, e_{2}=\frac{\partial}{\partial v}$. Since $e_{1}=\frac{\partial}{\partial u}$ and $e_{2}=\frac{\partial}{\partial v}$ are orthonormal, $x$ is a Lagrangian immersion, we get

$$
\left\langle x_{u}, x_{u}\right\rangle=\left\langle x_{v}, x_{v}\right\rangle=1,\left\langle x_{u}, x_{v}\right\rangle=\left\langle x_{u}, i x_{v}\right\rangle=0 .
$$


From $\nabla_{e_{i}} e_{j}=0, i, j=1,2$ and $h_{11}^{1^{*}}=\frac{\sqrt{2}}{2}, h_{22}^{1^{*}}=\frac{\sqrt{2}}{2}, h_{11}^{2^{*}}=0, h_{22}^{2^{*}}=0$, we have

$$
x_{u u}=x_{v v}=\frac{\sqrt{2} i}{2} x_{u}, x_{u v}=\frac{\sqrt{2} i}{2} x_{v} .
$$

The self-shrinker condition (1.1) and $|x|^{2}=2$ imply that

$$
\mathbf{H}=x_{u u}+x_{v v}=-x,\langle x, x\rangle=2 .
$$

Using (4.9)-(4.11), we obtain the following explicit expression of $x$.

$$
x(u, v)=e^{\frac{i u}{\sqrt{2}}}\left(a_{1} e^{\frac{i v}{\sqrt{2}}}+a_{2} e^{\frac{-i v}{\sqrt{2}}}, b_{1} e^{\frac{i v}{\sqrt{2}}}+b_{2} e^{\frac{-i v}{\sqrt{2}}}\right) \in \mathbb{C}^{2},
$$

where $a_{1}, a_{2}, b_{1}, b_{2}$ are constant complex numbers satisfying that $a_{1} \overline{a_{1}}+b_{1} \overline{b_{1}}=a_{2} \overline{a_{2}}+b_{2} \overline{b_{2}}=$ $1, a_{1} \overline{a_{2}}+b_{1} \overline{b_{2}}=0$. Therefore, up to an isometry of $\mathbb{C}^{2}, x$ is congruent with

$$
x(u, v)=e^{\frac{i u}{\sqrt{2}}}\left(e^{\frac{i v}{\sqrt{2}}}, e^{\frac{-i v}{\sqrt{2}}}\right) \in \mathbb{C}^{2} .
$$

We choose local coordinates $s, t$ such that $s=\frac{u+v}{\sqrt{2}}, t=\frac{u-v}{\sqrt{2}}$, then $x$ is congruent with

$$
x(s, t)=\left(e^{i s}, e^{i t}\right) \in \mathbb{C}^{2},
$$

which is the standard expression of the Clifford torus $\mathbb{S}^{1}(1) \times \mathbb{S}^{1}(1)$ in $\mathbb{C}^{2}$.

Proof of Theorem 1.2 : Under the assumptions of Theorem 1.2, from Lemma 4.1, we know that $|A|^{2}=2$, which means that $|A|^{2}$ is constant. Then applying our key Proposition 4.2, we obtain that $M^{2}$ is the Clifford torus $\mathbb{S}^{1}(1) \times \mathbb{S}^{1}(1)$.

Remark 4.3. If $x: M^{2} \rightarrow \mathbb{R}^{3}$ is a compact orientable embedded self-shrinker with $|A|^{2} \leq 2$, then it follows from Gauss equation and Gauss-Bonnet theorem that

$$
8 \pi\left(1-\operatorname{gen}\left(M^{2}\right)\right)=2 \int_{M}^{2} K d v=\int_{M}^{2}\left(H^{2}-|A|^{2}\right) d v=\int_{M}^{2}\left(2-|A|^{2}\right) d v,
$$

where gen $\left(M^{2}\right)$ stands for the genus of $M^{2}, K$ is the Gauss curvature of $M^{2}$ and the last equality is due to the following identity by using the self-shrinker equation (1.1):

$$
\frac{1}{2} \Delta\left(|x|^{2}\right)=2+\langle x, \Delta x\rangle=2+\langle x, \mathbf{H}\rangle=2-H^{2} .
$$

From $|A|^{2} \leq 2$ it follows that either (i) the genus of $M^{2}$ is 0 , or (ii) the genus of $M^{2}$ is 1 and $|A|^{2}=2$. If the genus of $M^{2}$ is 0, then Brendle's result (see [3], Theorem 1) implies that $M^{2}$ is the round sphere $\mathbb{S}^{2}(\sqrt{2})$. If $M^{2}$ is a 2-dimensional closed self-shrinker in $\mathbb{R}^{3}$ satisfying that $|A|^{2}$ is constant, then Ding and Xin's result (see Theorem 4.2 of [10], see also [11] for a new proof) implies that $|A|^{2}=1$. Therefore, case (ii) the genus of $M^{2}$ is 1 and $|A|^{2}=2$ can not occur. So we obtain the following new characterization of the round sphere as a self-shrinker.

Proposition 4.4. Let $x: M^{2} \rightarrow \mathbb{R}^{3}$ be a compact orientable embedded self-shrinker. If $|A|^{2} \leq 2$, then $|A|^{2}=1$ and $M^{2}$ is the round sphere $\mathbb{S}^{2}(\sqrt{2})$.

\section{Proof of Theorem 1.4}

In this section, we prove Theorem 1.4. We also prove that a compact orientable Lagrangian self-shrinker in $\mathbb{C}^{2}$ with constant Gauss curvature must be the Riemannian product of two closed Abresch-Langer curves. As an application, we obtain several new characterizations of the Clifford torus as a Lagrangian self-shrinker in $\mathbb{C}^{2}$.

Lemma 5.1. Let $x: M^{2} \rightarrow \mathbb{C}^{2}$ be a compact orientable Lagrangian self-shrinker. If the Gauss curvature $K$ of $M^{2}$ is nonnegative, then $K=0$ and $M^{2}$ is a topological torus. 
Proof. Using the fact that there exist no Lagrangian self-shrinkers in $\mathbb{C}^{n}$ with the topology of sphere,which was proved by Smoczyk (see [23], Theorem 2.3.5, see also Theorem 2.1 in [6] for a detailed proof), we get gen $\left(M^{2}\right) \geq 1$, where gen $\left(M^{2}\right)$ stands for the genus of $M^{2}$. From Gauss-Bonnet theorem, we derive

$$
4 \pi\left(1-\operatorname{gen}\left(M^{2}\right)\right)=\int_{M}^{2} K d v
$$

If $K \geq 0$, then $\operatorname{gen}\left(M^{2}\right) \leq 1$. Hence, if $K \geq 0$, then $K=0$ and $M^{2}$ is a topological torus.

Proposition 5.2. Let $x: M^{2} \rightarrow \mathbb{C}^{2}$ be a compact orientable Lagrangian self-shrinker. If the Gauss curvature $K$ of $M^{2}$ is constant, then $K=0$ and $M^{2}$ is a topological torus.

Proof. First, using Lemma 5.1, if $K \geq 0$, then $K=0$. Hence, if $K$ is constant, then $K \leq 0$. Next, we prove that $K=0$. It follows from (3.3) and $\mathcal{L}=\Delta-\langle x, \nabla \cdot\rangle$ that

$$
\begin{aligned}
\frac{1}{2} \mathcal{L}|\mathbf{H}|^{2} & =\frac{1}{2} \mathcal{L} H^{2}=\sum_{k, i}\left(H_{, i}^{k^{*}}\right)^{2}+H^{2}-\sum_{i, j, k, l} H^{k^{*}} H^{l^{*}} h_{i j}^{k^{*}} h_{i j}^{l^{*}} \\
& =\left|\nabla^{\perp} \mathbf{H}\right|^{2}+H^{2}-\sum_{i, j, k, l} H^{k^{*}} H^{l^{*}} h_{i j}^{k^{*}} h_{i j}^{l^{*}},
\end{aligned}
$$

on the other hand, from Lemma 3.2 we know that

$$
\frac{1}{2} \mathcal{L}|A|^{2}=|\nabla A|^{2}+|A|^{2}-\frac{3}{2}|A|^{4}+2 H^{2}|A|^{2}-\frac{1}{2} H^{4}-\sum_{i, j, k, l} H^{k^{*}} H^{l^{*}} h_{i j}^{k^{*}} h_{i j}^{l^{*}} .
$$

Therefore, using Gauss equation $2 K=H^{2}-|A|^{2}$, we derive

$$
\mathcal{L} K=\left|\nabla^{\perp} \mathbf{H}\right|^{2}-|\nabla A|^{2}+H^{2}-\left(|A|^{2}-\frac{3}{2}|A|^{4}+2 H^{2}|A|^{2}-\frac{1}{2} H^{4}\right) .
$$

As $M^{2}$ is compact, there exists a point $p_{0} \in M^{2}$ such that $|x|^{2}$ attains its maximum at $p_{0}$. We immediately have $\left(|x|^{2}\right)_{, j}=0,1 \leq j \leq 2$ at $p_{0}$, which implies that $\left\langle x, e_{j}\right\rangle\left(p_{0}\right)=$ $0,1 \leq j \leq 2$. Hence at $p_{0}$, from (3.1) and (3.2) we have $x=-\mathbf{H},|x|^{2}=H^{2}, H_{i}^{k^{*}}=$ $0,1 \leq i, k \leq 2$. On the other hand, since $K$ is constant, $K_{, k} \equiv 0,1 \leq k \leq 2$. Using Gauss equation $2 K=H^{2}-|A|^{2}$, we get that $\left(|A|^{2}\right)_{, k}=0,1 \leq k \leq 2$ at $p_{0}$. Hence, (4.2) and (4.3) hold at $p_{0}$. Using the same argument as in the proof of Proposition 4.2, we have the following two possibilities:

(i) At $p_{0}, h_{11}^{1^{*}}=3 h_{22}^{1^{*}}, h_{22}^{2^{*}}=3 h_{11}^{2^{*}}$. In this case, $|A|^{2}\left(p_{0}\right)=\frac{4}{3}\left(\left(h_{11}^{1^{*}}\right)^{2}+\left(h_{22}^{2^{*}}\right)^{2}\right), H^{2}\left(p_{0}\right)=$ $\frac{16}{9}\left(\left(h_{11}^{1^{*}}\right)^{2}+\left(h_{22}^{2^{*}}\right)^{2}\right)$, so we get $|A|^{2}\left(p_{0}\right)=\frac{3}{4} H^{2}\left(p_{0}\right), 0 \geq K=K\left(p_{0}\right)=\frac{1}{2}\left(H^{2}\left(p_{0}\right)-\right.$ $\left.|A|^{2}\left(p_{0}\right)\right)=\frac{1}{8} H^{2}\left(p_{0}\right) \geq 0$, which implies that $K=0$.

(ii) At $p_{0}, h_{11,1}^{1^{*}}=h_{11,2}^{1^{*}}=h_{22,1}^{1^{*}}=h_{22,2}^{1^{*}}=h_{22,2}^{2^{*}}=0$. In this case, $|\nabla A|=0$ at $p_{0}$. Since $K$ is constant, we get $\mathcal{L} K \equiv 0$, then at $p_{0}$, from (5.4) we have

$$
H^{2}-\left(|A|^{2}-\frac{3}{2}|A|^{4}+2 H^{2}|A|^{2}-\frac{1}{2} H^{4}\right)=\frac{1}{2}\left(H^{2}-3|A|^{2}+2\right)\left(H^{2}-|A|^{2}\right)=0,
$$

from which we deduce that either $H^{2}\left(p_{0}\right)=3|A|^{2}\left(p_{0}\right)-2$ or $H^{2}\left(p_{0}\right)=|A|^{2}\left(p_{0}\right)$. If $H^{2}\left(p_{0}\right)=3|A|^{2}\left(p_{0}\right)-2$, then $K=K\left(p_{0}\right)=\frac{1}{2}\left(H^{2}\left(p_{0}\right)-|A|^{2}\left(p_{0}\right)\right)=\frac{1}{3}\left(H^{2}\left(p_{0}\right)-1\right) \leq 0$, so we get $H^{2}\left(p_{0}\right) \leq 1$. On the other hand, since $|x|^{2}$ attains its maximum at $p_{0}$, from Lemma 3.3. we deduce that $H^{2}\left(p_{0}\right)=|x|^{2}\left(p_{0}\right) \geq 2$, which contradicts with $H^{2}\left(p_{0}\right) \leq 1$. So we get $H^{2}\left(p_{0}\right)=|A|^{2}\left(p_{0}\right)$, which implies that $K=K\left(p_{0}\right)=0$.

Therefore, we have proved that $K=0$. It follows from Gauss-Bonnet theorem that $M^{2}$ is a topological torus. 
Proposition 5.3. Let $x: M^{2} \rightarrow \mathbb{C}^{2}$ be a Lagrangian self-shrinker. If $M^{2}$ is flat, then $M^{2}$ is locally an open part of the Riemannian product of two Abresch-Langer curves.

Proof. We define $U_{1}=\left\{p \in M^{2} \mid p\right.$ is a totally geodesic point $\}, U_{2}=M^{2}-U_{1}$. If $p \in M^{2}$ is an interior point of $U_{1}$, then $M^{2}$ is locally the Riemannian product of two straight lines around $p$ (straight line is also a special Abresch-Langer curve).

In the following, without loss of generality, we assume that $p \in U_{2}$, i.e., $p$ is not a totally geodesic point. We denote $U M_{p}=\left\{u \in T_{p} M^{2}|| u \mid=1\right\}$, define $f(u)=\langle h(u, u), J u\rangle(u \in$ $\left.U M_{p}\right)$ and take $e_{1}$ as a vector in which $f$ attains its maximum. We choose $e_{2} \in T_{p} M^{2}$ as a unit vector which is orthogonal to $e_{1}$. As $f$ attains its maximum in $e_{1}$, we immediately have $\left\langle h\left(e_{1}, e_{1}\right), J e_{2}\right\rangle=0$, which implies that there exists a number $\lambda_{1}>0$ such that $h\left(e_{1}, e_{1}\right)=\lambda_{1} J e_{1}$. Since $\langle h(X, Y), J Z\rangle$ is totally symmetric (see (2.3)), there exist two numbers $\lambda_{0}$ and $\lambda_{2}$ such that

$$
h\left(e_{1}, e_{2}\right)=\lambda_{0} J e_{2}, h\left(e_{2}, e_{2}\right)=\lambda_{0} J e_{1}+\lambda_{2} J e_{2} .
$$

Moreover, since $f$ attains its maximum in $e_{1}$, we have $\lambda_{1} \geq 2 \lambda_{0}$, and if $\lambda_{1}=2 \lambda_{0}$, then $\lambda_{2}=0$ (see Lemma 1 in [19]). As $M^{2}$ is flat, from Gauss equation we have

$$
0=\left\langle h\left(e_{1}, e_{1}\right), h\left(e_{2}, e_{2}\right)\right\rangle-\left\langle h\left(e_{1}, e_{2}\right), h\left(e_{1}, e_{2}\right)\right\rangle=\lambda_{0}\left(\lambda_{1}-\lambda_{0}\right) .
$$

We claim that $\lambda_{0}=0$, if not, $0<\lambda_{1}=\lambda_{0}$ which contradicts with $\lambda_{1} \geq 2 \lambda_{0}$. So we obtain an orthonormal basis $e_{1}, e_{2}$ at $p$ such that

$$
h\left(e_{1}, e_{1}\right)=\lambda_{1} J e_{1}, h\left(e_{1}, e_{2}\right)=0, h\left(e_{2}, e_{2}\right)=\lambda_{2} J e_{2} .
$$

Next, we prove that there exists a neighborhood $U$ of $p$, local orthonormal vector fields $E_{1}, E_{2}$ and local functions $\Lambda_{1}, \Lambda_{2}$ such that at each point $q \in U$, we have

$$
h\left(E_{1}(q), E_{1}(q)\right)=\Lambda_{1}(q) J E_{1}(q), h\left(E_{1}(q), E_{2}(q)\right)=0, h\left(E_{2}(q), E_{2}(q)\right)=\Lambda_{2}(q) J E_{2}(q) .
$$

We choose an arbitrary orthonormal vector field $F_{1}, F_{2}$ in a neighborhood $V$ of $p$ such that $F_{i}(p)=e_{i}$. We denote $h_{i j}^{k}(q)=\left\langle h\left(F_{i}(q), F_{j}(q)\right), J F_{k}(q)\right\rangle(\forall q \in V)$ and consider the following system of equations:

$$
\left\{\begin{array}{l}
L_{1}\left(y^{1}(q), y^{2}(q), \Lambda_{1}(q)\right):=\sum_{j, k} h_{j k}^{1}(q) y^{j}(q) y^{k}(q)-y^{1}(q) \Lambda_{1}(q)=0 \\
L_{2}\left(y^{1}(q), y^{2}(q), \Lambda_{1}(q)\right):=\sum_{j, k} h_{j k}^{2}(q) y^{j}(q) y^{k}(q)-y^{2}(q) \Lambda_{1}(q)=0 \\
L_{3}\left(y^{1}(q), y^{2}(q), \Lambda_{1}(q)\right):=\left(y^{1}(q)\right)^{2}+\left(y^{2}(q)\right)^{2}-1=0 .
\end{array}\right.
$$

If we denote $L=\left(L_{1}, L_{2}, L_{3}\right), Y=\left(y^{1}, y^{2}, \Lambda_{1}\right)$, then $Y(p)=\left(1,0, \lambda_{1}\right)$ is a solution to $L(p)=0$, and

$$
\left.\left(\frac{\partial L_{i}}{\partial Y_{j}}\right)\right|_{p}=\left(\begin{array}{ccc}
\lambda_{1} & 0 & -1 \\
0 & -\lambda_{1} & 0 \\
2 & 0 & 0
\end{array}\right)
$$

is non-degenerate. Applying Implicit Function Theorem, there exists a unique smooth function $Y(q)=\left(y^{1}(q), y^{2}(q), \Lambda_{1}(q)\right)$ satisfying (5.8) in an open set $V_{1} \subset V$, with initial value $Y(p)=\left(1,0, \lambda_{1}\right)$. If we define $E_{1}(q)=\sum_{i=1}^{2} y^{i}(q) F_{i}(q)$, then (5.8) implies that $E_{1}$ is a smooth unit vector field in $V_{1}$ and $h\left(E_{1}(q), E_{1}(q)\right)=\Lambda_{1}(q) J E_{1}(q), \forall q \in V_{1}$.

Assume that $E_{2}$ is a smooth unit vector field in $V_{1}$ such that $E_{1}$ and $E_{2}$ are orthogonal, using the property that $\langle h(X, Y), J Z\rangle$ is totally symmetric (see (2.3) ), we get that there 
exist two local functions $\Lambda_{0}$ and $\Lambda_{2}$ such that

$$
h\left(E_{1}, E_{2}\right)=\Lambda_{0} J E_{2}, h\left(E_{2}, E_{2}\right)=\Lambda_{0} J E_{1}+\Lambda_{2} J E_{2}, \forall q \in V_{1} .
$$

As $\left(\Lambda_{1}-\Lambda_{0}\right)(p)=\lambda_{1}-\lambda_{0}=\lambda_{1}-0>0$, there exists an open set $U \subset V_{1}$ such that

$$
\left(\Lambda_{1}-\Lambda_{0}\right)(q)>0, \forall q \in U \text {. }
$$

Moreover, since $M^{2}$ is flat, from Gauss equation we have

$$
0=\left\langle h\left(E_{1}, E_{1}\right), h\left(E_{2}, E_{2}\right)\right\rangle-\left\langle h\left(E_{1}, E_{2}\right), h\left(E_{1}, E_{2}\right)\right\rangle=\Lambda_{0}\left(\Lambda_{1}-\Lambda_{0}\right),
$$

so we derive $\Lambda_{0}=0, \forall q \in U$. Therefore, we have found a neighborhood $U$ of $p$, local orthonormal vector fields $E_{1}, E_{2}$ and local functions $\Lambda_{1}, \Lambda_{2}$ such that at each point $q \in U$, (5.7) is satisfied.

In the following, we use Codazzi equations and the self-shrinker equation to deduce that $x$ is locally a product immersion. As $E_{1}$ and $E_{2}$ are local orthonormal tangent vector fields, we can write the covariant derivatives as follows.

$$
\nabla_{E_{1}} E_{1}=\alpha E_{2}, \nabla_{E_{1}} E_{2}=-\alpha E_{1}, \nabla_{E_{2}} E_{1}=-\beta E_{2}, \nabla_{E_{2}} E_{2}=\beta E_{1},
$$

where $\alpha$ and $\beta$ are local functions. It follows from (5.7), (5.10) and the Codazzi equation $\left(\nabla_{E_{1}} h\right)\left(E_{2}, E_{2}\right)=\left(\nabla_{E_{2}} h\right)\left(E_{1}, E_{2}\right)$ that

$$
\begin{gathered}
\alpha \Lambda_{2}-\beta \Lambda_{1}=0, \\
E_{1}\left(\Lambda_{2}\right)=\beta \Lambda_{2} .
\end{gathered}
$$

If we denote $x^{T}=x-x^{\perp}$, then $x^{T}$ is the tangent part of the position vector $x$. By using (2.1), (2.2), (2.3), (5.7) and (5.10), we derive

$$
\left\{\begin{array}{l}
\left\langle D_{E_{1}}\left(-x^{\perp}\right), J E_{2}\right\rangle=\left\langle D_{E_{1}}\left(x^{T}-x\right), J E_{2}\right\rangle=\left\langle D_{E_{1}}\left(x^{T}\right)-D_{E_{1}} x, J E_{2}\right\rangle \\
=\left\langle D_{E_{1}}\left(x^{T}\right)-E_{1}, J E_{2}\right\rangle=\left\langle D_{E_{1}}\left(x^{T}\right), J E_{2}\right\rangle=\left\langle\nabla_{E_{1}}\left(x^{T}\right)+h\left(E_{1}, x^{T}\right), J E_{2}\right\rangle \\
=\left\langle h\left(E_{1}, x^{T}\right), J E_{2}\right\rangle=\left\langle h\left(E_{1}, E_{2}\right), J x^{T}\right\rangle=\left\langle 0, J x^{T}\right\rangle=0, \\
\left\langle D_{E_{1}} \mathbf{H}, J E_{2}\right\rangle=\left\langle\nabla_{E_{1}}^{\perp} \mathbf{H}, J E_{2}\right\rangle=\left\langle\nabla_{E_{1}}^{\perp}\left(\Lambda_{1} J E_{1}+\Lambda_{2} J E_{2}\right), J E_{2}\right\rangle=E_{1}\left(\Lambda_{2}\right)+\alpha \Lambda_{1},
\end{array}\right.
$$

which combined with the self-shrinker equation (1.1) $\left(\mathbf{H}=-x^{\perp}\right)$ imply

$$
E_{1}\left(\Lambda_{2}\right)+\alpha \Lambda_{1}=0 .
$$

From (5.12) and (5.13) we derive

$$
\alpha \Lambda_{1}+\beta \Lambda_{2}=0
$$

Taking the sum of the square of (5.11) and the square of (5.14), we derive

$$
\left(\alpha^{2}+\beta^{2}\right)\left(\Lambda_{1}^{2}+\Lambda_{2}^{2}\right)=0
$$

since $\Lambda_{1}>0$ on $U$, we conclude that $\alpha=\beta=0$ on $U$, which means that $E_{1}$ and $E_{2}$ are both totally geodesic distributions on $U$. Therefore, applying the theorem of Frobenius, there exist local coordinates $\{s, t\}$ on $U$ such that $E_{1}=\frac{\partial}{\partial s}, E_{2}=\frac{\partial}{\partial t}$, and $M^{2}$ is locally a Riemannian product $I_{1} \times I_{2} \in \mathbb{R} \times \mathbb{R}$. Since the second fundamental form satisfies (5.7), using a lemma of J. D. Moore (see Lemma in the end of section 2 of [20]), we know that $x$ is locally a product immersion. Here we present a direct proof of this conclusion. Since $E_{1}=\frac{\partial}{\partial s}$ and $E_{2}=\frac{\partial}{\partial t}$ are orthonormal, $x$ is a Lagrangian immersion, we derive

$$
\left\langle x_{s}, x_{s}\right\rangle=\left\langle x_{t}, x_{t}\right\rangle=1,\left\langle x_{s}, x_{t}\right\rangle=\left\langle x_{s}, i x_{t}\right\rangle=0 .
$$

From (5.7) and (5.10), using $\alpha=\beta=0$, we have

$$
x_{s t}=0,
$$


which implies that there exist four complex functions $f_{i}(s), g_{i}(t), i=1,2$ such that

$$
x=\left(f_{1}(s)+g_{1}(t), f_{2}(s)+g_{2}(t)\right) \in \mathbb{C}^{2} .
$$

(5.18) combined with (5.16) imply

$$
\left\{\begin{array}{l}
\left|f_{1}^{\prime}(s)\right|^{2}+\left|f_{2}^{\prime}(s)\right|^{2}=1,\left|g_{1}^{\prime}(t)\right|^{2}+\left|g_{2}^{\prime}(t)\right|^{2}=1, \\
\left|f_{1}^{\prime}(s)\right|^{2}+\left|g_{1}^{\prime}(t)\right|^{2}=1,\left|f_{2}^{\prime}(s)\right|^{2}+\left|g_{2}^{\prime}(t)\right|^{2}=1, \\
f_{1}^{\prime}(s){\overline{g_{1}}}^{\prime}(t)+f_{2}^{\prime}(s){\overline{g_{2}}}^{\prime}(t)=0, \\
f_{1}^{\prime}(s){\overline{f_{2}}}^{\prime}(s)+g_{1}^{\prime}(t){\overline{g_{2}}}^{\prime}(t)=0 .
\end{array}\right.
$$

In (5.19), the equations in the first and third lines are direct consequences of (5.18) combined with (5.16) and these equations mean that the matrix $A=\left[\begin{array}{cc}f_{1}^{\prime}(s) & f_{2}^{\prime}(s) \\ g_{1}^{\prime}(t) & g_{2}^{\prime}(t)\end{array}\right]$ is a unitary matrix, so we obtain the equations in the second and forth lines of (5.19). that

Using (5.19), there exist two real constants $\theta_{0}, \theta_{1}$ and two real functions $f(s), g(t)$ such

$$
f_{1}^{\prime}(s)=\cos \theta_{0} e^{i f(s)}, f_{2}^{\prime}(s)=\sin \theta_{0} e^{i \theta_{1}} e^{i f(s)}, g_{1}^{\prime}(t)=-\sin \theta_{0} e^{i g(t)}, g_{2}^{\prime}(t)=\cos \theta_{0} e^{i \theta_{1}} e^{i g(t)} .
$$

If we denote $F(s)=\int_{0}^{s} e^{i f(\tilde{s})} d \tilde{s}, G(t)=\int_{0}^{t} e^{i g(\tilde{t})} d \tilde{t}$, then we obtain

$$
x=\left(\cos \theta_{0} F(s)-\sin \theta_{0} G(t)+c_{1}, \sin \theta_{0} e^{i \theta_{1}} F(s)+\cos \theta_{0} e^{i \theta_{1}} G(t)+c_{2}\right),
$$

where $c_{1}$ and $c_{2}$ are two complex constants. By solving

$$
\cos \theta_{0} a_{1}-\sin \theta_{0} a_{2}=c_{1}, \sin \theta_{0} e^{i \theta_{1}} a_{1}+\cos \theta_{0} e^{i \theta_{1}} a_{2}=c_{2},
$$

we get a unique solution for $a_{1}$ and $a_{2}$, so $x$ can be expressed as

$$
x=\left(\cos \theta_{0}\left(F(s)+a_{1}\right)-\sin \theta_{0}\left(G(t)+a_{2}\right), \sin \theta_{0} e^{i \theta_{1}}\left(F(s)+a_{1}\right)+\cos \theta_{0} e^{i \theta_{1}}\left(G(t)+a_{2}\right)\right),
$$

where $a_{1}$ and $a_{2}$ are two complex constants. Therefore, up to an isometry of $\mathbb{C}^{2}, x$ is locally congruent with

$$
x(s, t)=\left(x_{1}(s), x_{2}(t)\right)=\left(F(s)+a_{1}, G(t)+a_{2}\right) \in \mathbb{C}^{2},
$$

which is locally a product immersion from a Riemannian product $I_{1} \times I_{2}$ to $\mathbb{C}^{2}$. Finally, since $M^{2}$ is a self-shrinker, from the self-shrinker equation (1.1), we obtain that $x_{1}(s)$ : $I_{1} \rightarrow \mathbb{C}$ and $x_{2}(t): I_{2} \rightarrow \mathbb{C}$ also satisfy the self-shrinker equation (1.1), hence we obtain that $M^{2}$ is locally an open part of the Riemannian product of two one-dimensional selfshrinkers in $\mathbb{C}=\mathbb{R}^{2}$, i.e., $M^{2}$ is locally an open part of the Riemannian product of two Abresch-Langer curves.

There is a special property of the Abresch-Langer curves (see Theorem A in [1] and Lemma 5.3 in [24]):

Lemma 5.4 (see Lemma 5.3 in [24]). If $x: \Gamma \rightarrow \mathbb{R}^{2}$ is an Abresch-Langer curve, $k$ is the curvature of $\Gamma$ with respect to its inner unit normal, then there exists a constant $c_{\Gamma}$ such that $k e^{-|x|^{2} / 2}=c_{\Gamma}$ holds on all of $\Gamma$. If $\Gamma_{1}, \Gamma_{2}$ are two Abresch-Langer curves with $c_{\Gamma_{1}}=c_{\Gamma_{2}}$, then up to a Euclidean motion $\Gamma_{1}=\Gamma_{2}$. Moreover, $k_{\min }$ and $k_{\max }$ satisfy $k_{\min } e^{-k_{\min }^{2} / 2}=k_{\max } e^{-k_{\max }^{2} / 2}=c_{\Gamma}$, hence $k_{\min }(\Gamma)>0$ if $\Gamma$ is not a straight line through the origin.

Applying Lemma 5.4, we know that if two Abresch-Langer curves $\Gamma_{1}$ and $\Gamma_{2}$ coincide on an open set, then $\Gamma_{1}$ and $\Gamma_{2}$ coincide completely. Consequently, if the Riemannian product of two Abresch-Langer curves $\left(x: \Gamma_{1} \times \Gamma_{2} \rightarrow \mathbb{C}^{2}\right)$ and the Riemannian product of other two Abresch-Langer curves $\left(\tilde{x}: \tilde{\Gamma_{1}} \times \tilde{\Gamma_{2}} \rightarrow \mathbb{C}^{2}\right)$ coincide on an open set, then $\Gamma_{1} \times \Gamma_{2}$ 
and $\tilde{\Gamma_{1}} \times \tilde{\Gamma_{2}}$ coincide completely. Using Lemma 5.4 and Proposition 5.3 , we conclude that Proposition 5.3 is also true in the global sense.

Proposition 5.5. Let $x: M^{2} \rightarrow \mathbb{C}^{2}$ be a complete connected Lagrangian self-shrinker. If $M^{2}$ is flat, then $M^{2}$ is the Riemannian product of two Abresch-Langer curves.

Proof. We use the same notations as in Proposition 5.3 .

We define $U_{1}=\left\{p \in M^{2} \mid p\right.$ is a totally geodesic point $\}, U_{2}=M^{2}-U_{1}$. It is obvious that $U_{1}$ is a closed set and $U_{2}$ is an open set. We prove that either $M^{2}=U_{1}$ or $M^{2}=U_{2}$. As $U_{2}$ is an open set, we immediately get that $U_{2}=\cup_{k} U_{2 k}$, where $U_{2 k}$ are open disjoint connected components of $U_{2}$. For any $k, \forall p \in U_{2 k}$, by using Proposition [5.3, we know that there exists a neighborhood $U_{p} \subset U_{2 k}$ such that $U_{p}$ is an open part of the Riemannian product of two Abresch-Langer curves. We denote $U_{2 k}=\cup_{p \in U_{2 k}} U_{p}$. If $p_{1}, p_{2} \in U_{2 k}, U_{p_{1}} \cap U_{p_{2}} \neq \emptyset$, $U_{p_{1}}$ is an open part of $\Gamma_{1} \times \Gamma_{2}, U_{p_{2}}$ is an open part of $\tilde{\Gamma}_{1} \times \tilde{\Gamma}_{2}$, then $\Gamma_{1} \times \Gamma_{2}$ and $\tilde{\Gamma}_{1} \times \tilde{\Gamma_{2}}$ coincide on the nonempty open set $U_{p_{1}} \cap U_{p_{2}}$, so we obtain that $\Gamma_{1} \times \Gamma_{2}$ and $\tilde{\Gamma_{1}} \times \tilde{\Gamma}_{2}$ coincide completely. This implies that each component $U_{2 k}$ is an open part of two Abresch-Langer curves $\Gamma_{1 k} \times \Gamma_{2 k}$. By definition of $U_{2}, \forall p \in U_{2 k}, p$ is not a totally geodesic point, without loss of generality, we assume that $\Gamma_{1 k}$ is not a straight line, then we get that $k\left(\Gamma_{1 k}\right) \geq k_{\min }\left(\Gamma_{1 k}\right)>0$, then by use of continuity, $\forall \tilde{p} \in \overline{U_{2 k}},|A|^{2}(\tilde{p}) \geq k_{\min }^{2}\left(\Gamma_{1 k}\right)>0$, so we deduce that $\tilde{p}$ is not a totally geodesic point, which means that $\overline{U_{2 k}} \subset U_{2}$. On the other hand, $U_{2 k}$ is a connected component of $U_{2}$, so we get $\overline{U_{2 k}}=U_{2 k}$, hence $U_{2 k}$ is open and closed, which implies that either $U_{2 k}=\emptyset$ or $U_{2 k}=M^{2}$. Therefore, there are two possibilities: (i) $U_{2 k}=\emptyset, \forall k$. In this case, $U_{2}=\emptyset$ and $M^{2}=U_{1}$; (ii) $\exists k$ s.t. $U_{2 k}=M^{2}$. In this case $U_{1}=\emptyset$ and $M^{2}=U_{2}$.

If $M^{2}=U_{1}$, then $M^{2}$ is totally geodesic. As $M^{2}$ is complete and connected, we obtain that $M^{2}$ is the Riemannian product of two straight lines (straight line is also a special Abresch-Langer curve).

If $M^{2}=U_{2}$, since $M^{2}$ is complete and connected, then from the arguments above, we get that $M^{2}$ is the Riemannian product of two Abresch-Langer curves.

Proof of Theorem 1.4 : Under the assumptions of Theorem 1.4, from Lemma 5.1, we know that $K=0$ and $M^{2}$ is a topological torus. Then applying Proposition 5.5, we obtain that $M^{2}$ is the Riemannian product of two Abresch-Langer curves.

Combing Proposition 5.2 and Proposition 5.5, we obtain

Proposition 5.6. Let $x: M^{2} \rightarrow \mathbb{C}^{2}$ be a compact orientable Lagrangian self-shrinker. If the Gauss curvature $K$ of $M^{2}$ is constant, then $K=0$ and $M^{2}$ is the Riemannian product of two closed Abresch-Langer curves.

If $x: M^{2} \rightarrow \mathbb{C}^{2}$ is embedded, using the result of Abresch-Langer which states that the only closed embedded self-shrinker in $\mathbb{R}^{2}$ is the circle, as immediate consequences of Proposition 5.6, we obtain

Corollary 5.7. The Clifford torus $\mathbb{S}^{1}(1) \times \mathbb{S}^{1}(1)$ is the unique compact orientable embedded Lagrangian self-shrinker in $\mathbb{C}^{2}$ with constant Gauss curvature.

Remark 5.8. By a theorem of Whitney, any compact (without boundary) orientable embedded Lagrangian surface $M^{2}$ in $\mathbb{C}^{2}$ has to be a topological torus. If we assume that $M^{2}$ is a compact orientable embedded Lagrangian self-shrinker in $\mathbb{C}^{2}$ with nonpositive Gauss curvature, then by using Gauss-Bonnet theorem we obtain $K=0$. Using Proposition 5.5 and the result of Abresch-Langer which states that the only closed embedded self-shrinker in $\mathbb{R}^{2}$ is the circle, we deduce the following characterization of the Clifford torus. 
NEW CHARACTERIZATIONS OF THE CLIFFORD TORUS AS A LAGRANGIAN SELF-SHRINKER 15

Corollary 5.9. The Clifford torus $\mathbb{S}^{1}(1) \times \mathbb{S}^{1}(1)$ is the unique compact orientable embedded Lagrangian self-shrinker in $\mathbb{C}^{2}$ with nonpositive Gauss curvature.

Acknowledgements: The authors would like to thank the referee for the very careful review and for providing a number of valuable comments and suggestions.

\section{REFERENCES}

[1] Uwe Abresch and Joel C. Langer, The normalized curve shortening flow and homothetic solutions. J. Differential Geom., 23 (1986), no. 2, 175-196.

[2] Henri Anciaux, Construction of Lagrangian self-similar solutions to the mean curvature flow in $C^{n}$. Geom. Dedicata, 120 (2006), 37-48.

[3] Simon Brendle, Embedded self-similar shrinkers of genus 0. Ann. of Math., 183 (2016), no. 2, 715-728.

[4] Huai-Dong Cao and Haizhong Li, A gap theorem for self-shrinkers of the mean curvature flow in arbitrary codimension. Calc. Var. Partial Differential Equations, 46 (2013), no. 3-4, 879-889.

[5] Ildefonso Castro and Ana M. Lerma, Hamiltonian stationary self-similar solutions for Lagrangian mean curvature flow in the complex Euclidean plane. Proc. Amer. Math. Soc., 138 (2010), no. 5, 1821-1832.

[6] Ildefonso Castro and Ana M. Lerma, The Clifford torus as a self-shrinker for the Lagrangian mean curvature flow. Int. Math. Res. Not., 2014, no. 6, 1515-1527.

[7] Qing-Ming Cheng and Yejuan Peng, Complete self-shrinkers of the mean curvature flow. Calc. Var. Partial Differential Equations, 52 (2015), no. 3-4, 497-506.

[8] Qing-Ming Cheng and Guoxin Wei, A gap theorem of self-shrinkers. Trans. Amer. Math. Soc., 367 (2015), no. 7, 4895-4915.

[9] Tobias H. Colding and William P. Minicozzi II, Generic mean curvature flow I: generic singularities. Ann. of Math., 175 (2012), no. 2, 755-833.

[10] Qi Ding and Yuanlong Xin, The rigidity theorems of self-shrinkers. Trans. Amer. Math. Soc., 366 (2014), no. 10, 5067-5085.

[11] Qiang Guang, Self-shrinkers with second fundamental form of constant length. arXiv:1405.4230

[12] Gerhard Huisken, Asymptotic behavior for singularities of the mean curvature flow. J. Differential Geom., 31 (1990), no. 1, 285-299.

[13] Gerhard Huisken, Local and global behaviour of hypersurfaces moving by mean curvature. Differential geometry: partial differential equations on manifolds (Los Angeles, CA, 1990), 175-191, Proc. Sympos. Pure Math., 54, Part 1, Amer. Math. Soc., Providence, RI, 1993.

[14] Nam Q. Le and Natasa Sesum, Blow-up rate of the mean curvature during the mean curvature flow and a gap theorem for self-shrinkers. Comm. Anal. Geom., 19 (2011), no. 4, 633-659.

[15] Yng-Ing Lee and Mu-Tao Wang, Hamiltonian stationary cones and self-similar solutions in higher dimension. Trans. Amer. Math. Soc., 362 (2010), no. 3, 1491-1503.

[16] Haizhong Li and Luc Vrancken, A basic inequality and new characterization of Whitney spheres in a complex space form. Israel J. Math., 146 (2005), 223-242.

[17] Haizhong Li and Xianfeng Wang, A differentiable sphere theorem for compact Lagrangian submanifolds in complex Euclidean space and complex projective space. Comm. Anal. Geom., 22 (2014), no. 2, 269288.

[18] Haizhong Li and Yong Wei, Classification and rigidity of self-shrinkers in the mean curvature flow. J. Math. Soc. Japan, 66 (2014), no. 3, 709-734.

[19] Sebastián Montiel and Francisco Urbano, Isotropic totally real submanifolds. Math. Z., 199 (1988), $55-60$.

[20] John Douglas Moore, Isometric immersions of Riemannian products. J. Differential Geom., 5 (1971), 159-168.

[21] André Neves, Singularities of Lagrangian mean curvature flow: monotone case. Math. Res. Lett., 17 (2010), no. 1, 109-126.

[22] André Neves, Recent progress on singularities of Lagrangian mean curvature flow. Surveys in geometric analysis and relativity, 413-438, Adv. Lect. Math. (ALM), 20, Int. Press, Somerville, MA, 2011.

[23] Knut Smoczyk, The Lagrangian mean curvature flow (Der Lagrangesche mittlere Krümmungsflu $\beta$ ). Leipzig: Univ. Leipzig (Habil.), 102 S. (2000).

[24] Knut Smoczyk, Self-shrinkers of the mean curvature flow in arbitrary codimension. Int. Math. Res. Not., 2005, no. 48, 2983-3004. 
Department of Mathematical Sciences, Tsinghua University, Beijing 100084, P. R. China E-mail address: hli@math.tsinghua.edu.cn

School of Mathematical Sciences and LPMC, Nankai University, Tianjin 300071, P. R. CHINA

E-mail address: wangxianfeng@nankai.edu.cn 